\title{
Clinical risk factors for loss of stent primary patency in patients with chronic legs ischemia
}

\author{
Klaudia Koza ${ }^{1, B-F}$, Paweł Grzelązka ${ }^{1, B, C, E, F}$, Adrianna Trofimiuk ${ }^{1, B, C, E, F}$, Karol Suppann 2, B, C, F, \\ Marcin Wasielewski², B, C, F, Joanna Wiśniewska ${ }^{2}, B, C, F$, Jacek Budzyński ${ }^{3, A-F}$ \\ ${ }^{1}$ Student Scientific Group, Chair and Clinic of Vascular and Internal Diseases, Faculty of Health Sciences, Ludwik Rydygier Collegium Medicum in Bydgoszcz, Nicolaus Copernicus \\ University in Toruń, Poland \\ ${ }^{2}$ Clinic of Vascular and Internal Diseases, Jan Biziel University Hospital No. 2, Bydgoszcz, Poland \\ ${ }^{3}$ Chair of Vascular and Internal Diseases, Faculty of Health Sciences, Ludwik Rydygier Collegium Medicum in Bydgoszcz, Nicolaus Copernicus University in Toruń, Poland \\ A - research concept and design; $\mathrm{B}$ - collection and/or assembly of data; $\mathrm{C}$ - data analysis and interpretation; \\ $D$ - writing the article; $E$ - critical revision of the article; $F$ - final approval of article
}

Address for correspondence

Jacek Budzyński

E-mail: budz@cps.pl

Funding sources

none declared

Conflict of interest

none declared

Received on May 30, 2015

Revised on June 24, 2015

Accepted on August 03, 2015

\begin{abstract}
Background. The outcome of endovascular therapy can be influenced by a number of factors, either demographic, biochemical, angiographic or procedural. Knowledge about these factors may help in the individualization of therapeutic methods, surveillance intensity, and should, ultimately, improve intervention efficacy.

Objectives. The aim of this study was to estimate the effect of clinical and biochemical factors on the late outcome of lower limb artery stenting.

Material and methods. The medical documentation of 91 patients with at least a 1 year follow-up after the stenting of a lower limb artery was retrospectively evaluated. Uni- and multivariate analyses were performed.

Results. Primary patency within an approximately 1.5-year follow-up amounted to 68.2\%. The probability of freedom from target lesion revascularization was significantly greater in patients with dyslipidemia. According to the Cox proportional-hazards analysis, the risk of target extremity revascularization was significantly affected by the following (hazard ratio [HR], 95\% confidence interval): Age $(0.93,0.88-0.99)$; dyslipidemia at inclusion (0.046, 0.01-0.23); LDL blood concentration (1.02; 1.01-1.04); hematocrit (1.2, 1.02-1.42); mean platelet volume $(0.66,0.44-0.99)$; INR (1.58, 1.13-2.21); and aPTT (1.18, 1.07-1.3).

Conclusions. Endovascular treatment with stenting in patients with atherosclerotic peripheral arterial disease is effective, but the risk of primary patency loss was affected by the presence of dyslipidemia, age, and blood coagulation parameters. The effect of dyslipidemia on stent failure occurrence should be evaluated in further studies.
\end{abstract}

Key words: risk factors, dyslipidemia, peripheral artery disease, in-stent restenosis, blood coagulation

DOI

$10.17219 /$ acem/58997

Copyright

Copyright by Author(s)

This is an article distributed under the terms of the

Creative Commons Attribution Non-Commercial License

(http://creativecommons.org/licenses/by-nc-nd/4.0/) 
The endovascular treatment of peripheral artery disease (PAD) is still progressing and is associated with many complications that reduce its later benefits, such as in-stent thrombosis, stent fracture, in-stent restenosis (ISR) and stent infection. ${ }^{1,2}$ A common factor affecting the outcome of endovascular therapy is atherosclerosis progression, which requires consecutive limb revascularization outside the primary treated site. One year after an endovascular procedure, the primary patency of a stent implanted into an iliac artery amounts to $81-94 \%$, and 60-82\% for stents implanted into a femoropopliteal segment of the vascular bed, depending on the indications for the procedure (claudication or critical limb ischemia, CLI) and lesion severity (stenosis or total occlusion). ${ }^{1,2}$

Advances in special equipment dedicated to endovascular therapy has helped to reduce the prevalence of the above-mentioned complications, and offer the possibility of treating stent failure. Among such techniques are the use of: a) percutaneous angioplasty using plain old balloon angioplasty (POBA), drug-eluting balloons (DEB), and cutting balloons or cryoplasty; b) different scaffold techniques with the use of bare metal stents (BMS), drug-eluting stents (DES), cytokine- or growth-factor-eluting stents, covered stents, and bare or drug-eluting bioresorbable vascular scaffolding (BVS); as well as c) debulking techniques (atherectomy, and turbo-booster and excimer lasers).,13-12 Few of these modern techniques are widely available, and many are also more aggressive and expensive. Therefore, they cannot be used for all patients. For this reason, the proper identification of patients with a high risk of loss of stent primary patency, which may be an effect of both atherosclerosis progression and smooth muscle (neointima) proliferation, may help in the individualization of patients' qualification for respective treatment methods and, in this way, improve percutaneous therapy outcome. Such patients might also have different surveillance patterns. However, the majority of papers focus mainly on the following predictors of the primary patency of stenting: angiographic lesion severity (stenosis or occlusion), procedural factors (indications for procedure [claudication vs critical limb ischemia], type of patency restored [subintimal or intraluminal], length of stent, and outflow state). ${ }^{2,3,11-14}$ For this reason, we analyzed the effect of clinical and biochemical factors on the outcome of lower limb artery stenting.

\section{Material}

The medical documentation of 91 consecutive patients treated endovascularly between 2008 and 2013 with lower extremity artery stenting due to atherosclerosis was retrospectively evaluated. The inclusion criteria were as follows: a) first-time stenting of lower extremity arteries due to chronic leg ischemia; b) complete follow-up, for at least 1 year with visits at $1,3,6$, and 12 months after the endovascular procedure, and then at least every 6 months.
The exclusion criteria were: a) stent occlusion due to thrombosis or fracture, b) lack of compliance with suggestion regarding a change in lifestyle and the taking of medicines, and c) surgical revascularization during follow-up after stenting.

Percutaneous procedures in aorto-iliac level were performed in 26 (28.6\%) patients. Among them 4 (15\%) had grade A of lesion severity according to TASC classification, 4 (15\%) grade B, 10 (38.5\%) grade C, and 8 (31.5\%) grade D. ${ }^{2}$ In femoro-popliteal segment interventions were done in 65 (71.4\%) individuals. Of them, 4 (6\%) patients had lesions in the severity of grade A, 14 (21\%) grade B, 20 (31\%) grade C, and 27 (42\%) grade D. Twenty five (27.5\%) patients with intervention in femoro-popliteal segment needed additional angioplasty in the arteries below the knee to improve run-off. One stent was implanted in 49/91 (54\%) subjects. Two stents were introduced in $10 / 26(38 \%)$ patients with the lesions in aorto-iliac segment, and 32/65 (49\%) individuals with femoro-popliteal intervention. Thirty percent of patients were treated due to critical limbs ischemia (Table 1).

Table 1. Clinical data of patients with and without TLR

\begin{tabular}{|l|c|c|l|}
\hline Parameter & $\begin{array}{c}\text { TLR } \\
n=29\end{array}$ & $\begin{array}{c}\text { TLR-free } \\
n=62\end{array}$ & p-value \\
\hline Age (years) & $63.2 \pm 9.5$ & $62.9 \pm 8.4$ & 0.86 \\
Gender (male, $\mathrm{n} \%)$ & $24(83 \%)$ & $49(79 \%)$ & 0.68 \\
\hline Critical limb ischemia (n, \%) & $12(41.4 \%)$ & $15(24.2 \%)$ & 0.078 \\
Smoking habit (n, \%) & $14(48 \%)$ & $33(53 \%)$ & 0.66 \\
Hypertension (n, \%) & $19(66 \%)$ & $44(71 \%)$ & 0.60 \\
Diabetes mellitus (n, \%) & $10(35 \%)$ & $16(26 \%)$ & 0.40 \\
Fasting glucose (mg/dL) & $106.4 \pm 35.7$ & $106.6 \pm 25.6$ & 0.98 \\
BMI (kg/m $\left.{ }^{2}\right)$ & $25.5 \pm 4.8$ & $28.5 \pm 4.8$ & 0.14 \\
History of CAD (n, \%) & $13(45 \%)$ & $25(42 \%)$ & 0.78 \\
History of stroke (n, \%) & $4(14 \%)$ & $5(8 \%)$ & 0.43 \\
History of CHF (n, \%) & $4(14 \%)$ & $12(20 \%)$ & 0.48 \\
LDL cholesterol (mg/dL) & $116.0 \pm 41.7$ & $124.8 \pm 38.9$ & 0.40 \\
Triglycerides (mg/dL) & $118.2 \pm 46.0$ & $145.1 \pm 69.6$ & 0.12 \\
Dyslipidemia (n, \%) & $16(55 \%)$ & $55(89 \%)$ & 0.0002 \\
Leucocytes count (g/L) & $9.1 \pm 2.6$ & $9.1 \pm 2.5$ & 0.93 \\
Hemoglobin (g/L) & $13.3 \pm 1.8$ & $13.5 \pm 1.7$ & 0.64 \\
Hematocrit (\%) & $39.7 \pm 4.9$ & $39.9 \pm 4.5$ & 0.88 \\
MCV (fl) & $88.3 \pm 5.8$ & $89.2 \pm 5.5$ & 0.52 \\
Platelets count (g/L) & $290.5 \pm 115.5$ & $271.6 \pm 112.6$ & 0.47 \\
MPV (fl) & $9.4 \pm 1.5$ & $9.5 \pm 1.3$ & 0.67 \\
Creatinine (mg/dL) & $1.04 \pm 0.23$ & $1.1 \pm 0.2$ & 0.77 \\
INR & $1.44 \pm 0.2$ & $1.04 \pm 0.2$ & 0.08 \\
aPTT (s) & $33.2 \pm 9.2$ & $30.5 \pm 5.0$ & 0.14 \\
\hline
\end{tabular}

TLR - target lesion revascularization; BMI - body mass index; CAD - coronary artery disease; CHF - congestive heart failure; LDL - low-density lipoprotein; MCV - mean corpuscular volume; MPV - mean platelet volume; INR - international normalized ratio; aPTT - activated partial thromboplastin time. Some data was also presented earlier. ${ }^{13}$ 


\section{Methods}

According to the reference data, the following parameters were taken into consideration for analysis: age, gender, symptoms, smoking habits, hypertension, dyslipidemia (defined as low-density lipoprotein [LDL] cholesterol blood concentration at admission for a first endovascular procedure $\geq 100 \mathrm{mg} / \mathrm{dL}$, and/or triglyceride blood concentration on admission for a first endovascular procedure $\geq 150 \mathrm{mg} / \mathrm{dL}$ in spite of hypolipemic therapy with statins), diabetes mellitus, history of coronary artery disease (CAD), percutaneous coronary intervention $(\mathrm{PCI})$, coronary artery bypass graft (CABG), past stroke, history of congestive heart failure $(\mathrm{CHF})$, chronic kidney disease with blood creatinine level of $>2 \mathrm{mg} / \mathrm{dL}$, weight, height, body mass index (BMI), bilateral anklebrachial index (ABI), claudication distance, and biochemical parameters (LDL cholesterol, triglycerides, leucocyte count, hemoglobin concentration, hematocrit, mean corpuscular volume, platelet count, mean platelet volume $[\mathrm{MPV}]$, creatinine, activated partial thromboplastin time [aPTT], prothrombin time expressed as an international normalized ratio [INR], fasting blood glucose, and blood group). ${ }^{15}$ The kind of pharmacotherapy recommended was also analyzed (aspirin, clopidogrel, angiotensin-converting enzyme inhibitor [ACEI], angiotensin receptor blocker [ARB], beta-adrenergic receptor blocker, calcium channel antagonist, statin, type of statin, ezetimibe, fenofibrate, enoxaparin, warfarin, and acenocoumarol). All subjects were treated with aspirin, statin, ACEI, and beta-blockers.

The patients were followed up 1, 3, 6 and 12 months after the procedure and then every 6 months. On each control visit, symptom severity and the result of color-coded duplex ultrasound examination were evaluated. The need for revascularization was considered when resting leg pain, or claudication limiting usual daily physical activity was accompanied by a reduction of vessel diameter by $70 \%$ or more (peak systolic velocity index $>2.4$ ) or complete vessel occlusion appeared.

\section{Main outcomes measured}

During the period between percutaneous procedure performance and February 18, 2014 (the date of the Bioethical Committee agreement), the following main effectiveness outcomes of the analysis were taken: freedom from target lesion revascularization (TLR), target extremity revascularization (TER), and target limb amputation (TLA). ${ }^{15}$ TLR was defined as repeat percutaneous (endovascular) revascularization for a lesion anywhere within the stent or the $5 \mathrm{~mm}$ border proximal or distal to the stent due to symptom recurrence and target lesion occlusion (mainly due to IRS). This was treated with angioplasty (POBA or DEB) or stent-into-stent implantation. TER was defined in the wider criteria than usual, as the revascularization of a previously stented lower limb both due to the target lesion or a new lesion at least $10 \mathrm{~mm}$ outside the proximal or distal border of the stent due to symptom recurrence (claudication impeding normal daily functioning of the patient or critical limb ischemia) and lesion diameter stenosis $\geq 50 \%$ (effect of atherosclerosis progression). ${ }^{15}$ TLA was defined as minor or major amputation of a previously stented limb during the follow-up period.

The safety outcomes were also analyzed retrospectively. They were established as a major adverse event (MAE) and were as follows: in-hospital TLA as a result of endovascular procedure complication, periprocedural acute coronary syndrome (ACS), stroke, CHF exacerbation, acute kidney injury (AKI), and death. TLA was defined as a minor or major amputation of a previously stented limb during hospitalization during which an endovascular procedure was performed. Post-procedural AKI was defined as an abrupt increase in creatinine blood concentration within 7 days after an endovascular procedure.

\section{Ethics}

The study protocol was approved by the local Bioethics Committee (agreement number KB139/2014). All procedures have been conducted in compliance with the Declaration of Helsinki.

\section{Statistical analysis}

A licensed version of StatSoft, Inc. (2011), STATISTICA (data analysis software system), v. 10 was used. The normal distribution of the study variables was checked using the Shapiro-Wilk test. The results were mainly presented as the mean \pm standard deviation (SD) or $n, \%$. The statistical significance of the differences between patients needing an end-point procedure and patients not requiring such treatment was verified using the unpaired student's t-test and Fisher's exact test (Table 1). The survival analysis for the 91 subjects was carried out using Cox's F test, the log-rank test in the Kaplan-Meier method for 2 groups, and Cox proportional-hazards analysis.

\section{Results}

The mean observation time amounted to $544 \pm 503$ days. Within this follow-up, 29/91 (31.9\%) of the subjects required TLR, and the primary stent patency amounted to $68.1 \%$. An additional 3 patients needed an endovascular intervention with regard to lesions localized outside the previously implanted stent. For this reason, 32 (35.2\%) of the patients required TER. Minor or major leg amputation was performed in $5(5 / 91 ; 5.5 \%)$ patients. The majority of these patients required TLR $(4 / 5,80 \%$; $=0.038)$. 
The mean period between the first endovascular procedure and TLR, TER, and leg amputation amounted to $221 \pm 304$ days, $264 \pm 368$ days, and $455 \pm 521$ days, respectively. The mean time between TLR and minor or major leg amputation amounted to $314 \pm 484$ days.

Ninety-one patients were included. Their demographic and clinical data in relation to end-point occurrence are presented in Table 1 . The only significant difference between patients who required TLR (Table 1), TER or leg amputation and those who were free from these safety end-points was a lower prevalence of dyslipidemia upon admission among the former (Table 1). Diabetes mellitus, hypertension, smoking or history of cardiovascular event did not affect significantly the prevalence of TLR (Table 1, Fig. 2). When an analysis on TLR occurrence was performed separately for patients with interventions concerning aorto-iliac or femoro-popliteal segments as well as the severity of TASC grade of lesions localized in respective vascular segments, the effect of investigated clinical and biochemical risk factors on TLR prevalence was statistically not significant. ${ }^{2}$

Information regarding the use of the Kaplan-Meier analysis for 2 groups and the Cox regression model showed a significant negative association between the between the duration of TLR-free period and the presence of dyslipidemia at admission, which reduced the risk of TLR by about 20 times (Fig. 1). The positive correlation between TLR occurrence and APTT and INR values on the day of the first endovascular procedure was found (Table 2). Initial BMI and MPV negatively affected the

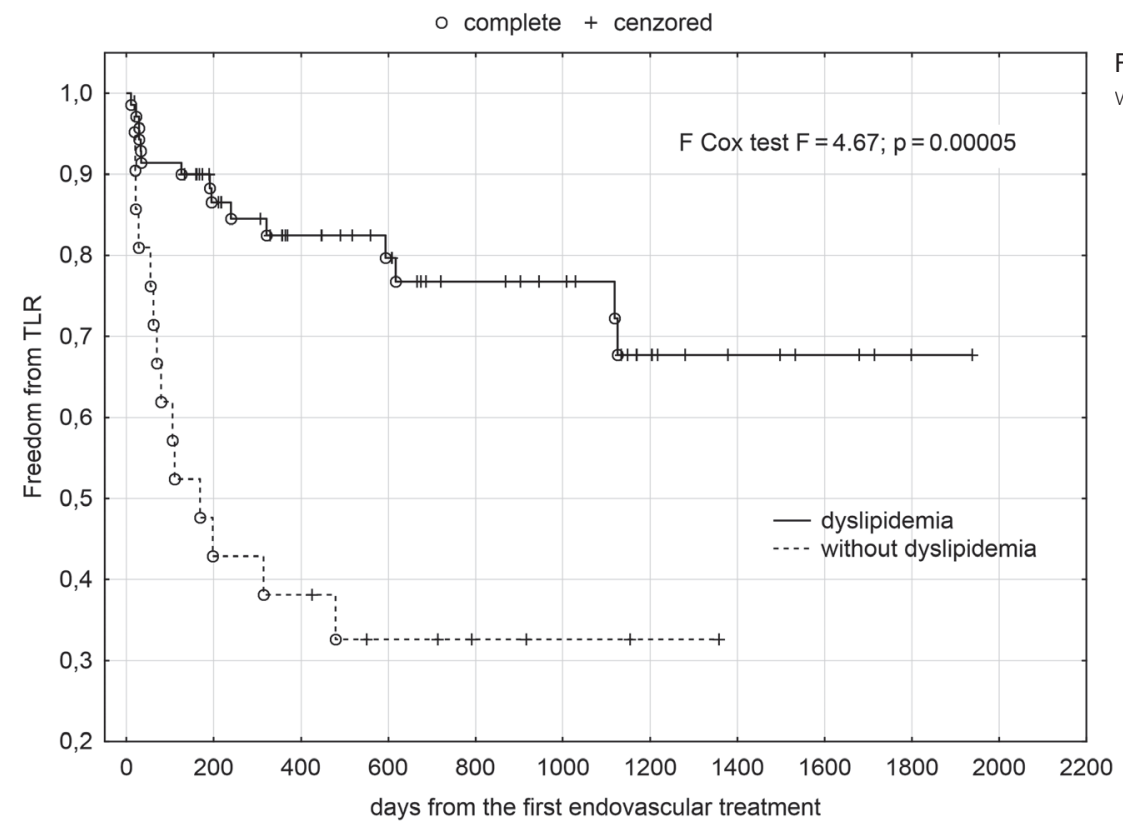

Fig. 1. Kaplan-Meier TLR-free survival curve in patients with and without dyslipidemia

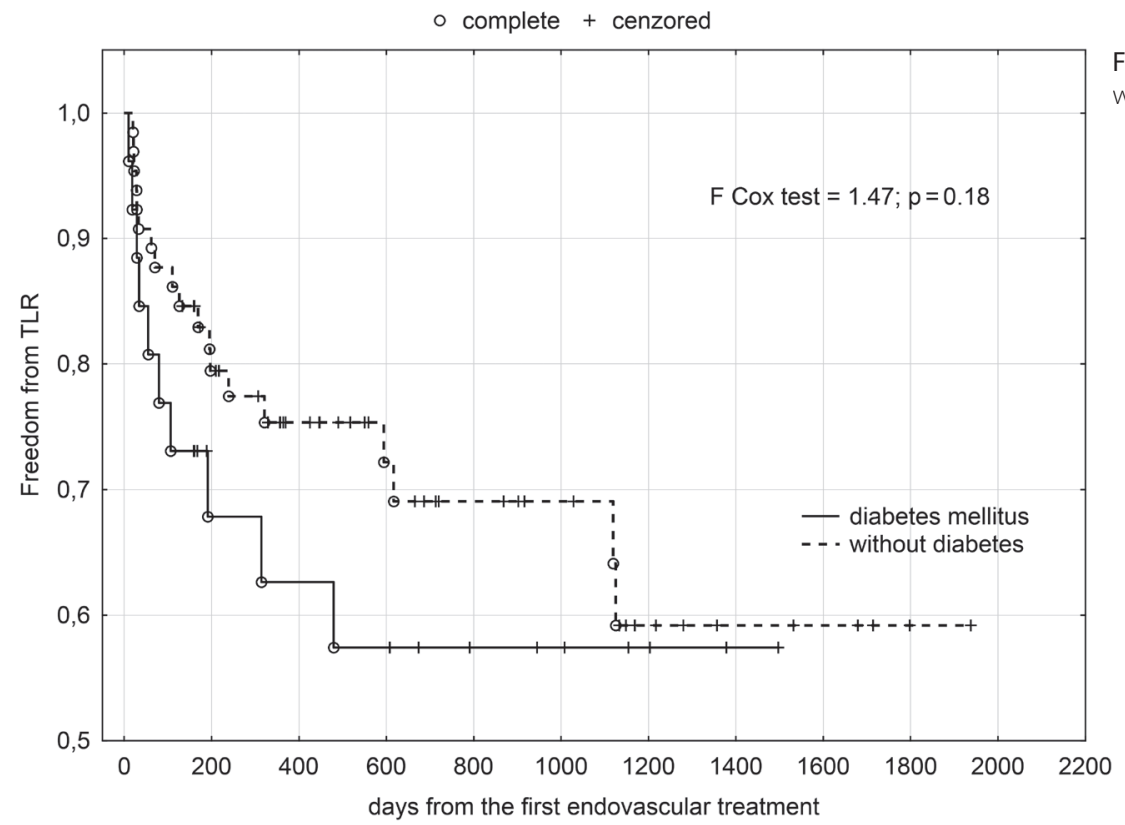

Fig. 2. Kaplan-Meier TLR-free survival curve in patients with and without diabetes mellitus 
Table 2. The Cox proportional-hazards regression model for target lesion revascularization (TLR). $\chi^{2}=43.4 ; p=0.0042$

\begin{tabular}{|c|c|c|c|c|c|}
\hline Parameter & $\beta$-coefficient & $\beta 95 \% \mathrm{Cl}$ & $\mathrm{p}$-value & $\begin{array}{c}\text { Hazard } \\
\text { ratio }\end{array}$ & $\begin{array}{c}\text { Hazard ratio } \\
95 \% \mathrm{Cl}\end{array}$ \\
\hline Age (years) & -0.06 & $-0.12-0.11$ & 0.11 & 0.95 & $0.88-1.01$ \\
\hline Gender (female/male) & 0.78 & $-0.71-2.26$ & 0.31 & 2.17 & $0.49-9.56$ \\
\hline Smoking & -0.46 & $-1.39-0.48$ & 0.34 & 0.63 & $0.25-1.61$ \\
\hline Hypertension & 0.38 & $-0.70-1.46$ & 0.49 & 1.46 & $0.49-4.3$ \\
\hline Diabetes mellitus & 0.81 & $-0.34-1.96$ & 0.17 & 2.25 & $0.71-7.11$ \\
\hline Dyslipidemia & -2.99 & $-4.65--1.34$ & 0.001 & 0.05 & $0.01-0.26$ \\
\hline BMI & -0.17 & $-0.36-0.02$ & 0.08 & 0.84 & $0.70-1.02$ \\
\hline History of CAD & -0.49 & $-1.67-0.68$ & 0.41 & 0.61 & $0.19-1.98$ \\
\hline Past stroke & -0.56 & $-2.15-1.03$ & 0.49 & 0.57 & $0.12-2.79$ \\
\hline History of $\mathrm{CHF}$ & -0.35 & $-2.16-1.47$ & 0.71 & 0.71 & $0.12-4.34$ \\
\hline Creatinine $>2 \mathrm{mg} / \mathrm{dL}$ & 1.66 & $-0.42-3.74$ & 0.12 & 5.27 & $0.66-42.22$ \\
\hline LDL cholesterol (mg/dL) & 0.01 & $-0.01-0.03$ & 0.21 & 1.01 & $0.99-1.03$ \\
\hline Triglycerides (mg/dL) & -0.01 & $-0.02-0.01$ & 0.60 & 0.99 & $0.98-1.01$ \\
\hline Leucocytes count (G/L) & -0.01 & $-0.12-0.1$ & 0.85 & 0.99 & $0.89-1.10$ \\
\hline Hematocrit (\%) & 0.11 & $-0.5-0.26$ & 0.18 & 1.11 & $0.95-1.3$ \\
\hline MCV & -0.03 & $-0.13-0.08$ & 0.64 & 0.97 & $0.87-1.08$ \\
\hline Platelets count (G/L) & -0.01 & $-0.01-0.01$ & 0.30 & 0.99 & $0.99-1.00$ \\
\hline MPV (fL) & -0.39 & $-0.82-0.05$ & 0.08 & 0.68 & $0.44-1.05$ \\
\hline Creatinine (mg/dL) & 1.65 & $-1.38-4.67$ & 0.29 & 5.19 & $0.25-106.8$ \\
\hline INR & 0.92 & $0.42-1.42$ & 0.001 & 2.52 & $1.52-4.16$ \\
\hline aPTT & 0.13 & $0.04-0.23$ & 0.005 & 1.14 & $1.04-1.25$ \\
\hline Fast glucose (mg/dL) & -0.01 & $-0.03-0.02$ & 0.62 & 0.99 & $0.97-1.02$ \\
\hline
\end{tabular}

$\mathrm{Cl}$ - confidence interval; BMI - body mass index; CAD - coronary artery disease; CHF - congestive heart failure; LDL - low-density lipoprotein; MCV - mean corpuscular volume; MPV - mean platelet volume; INR - international normalized ratio; aPTT - activated partial thromboplastin time.

length of freedom from TLR but only with borderline statistical significance (Table 2). Meanwhile, TER risk had a positive association with LDL blood concentration and aPTT and INR values, as well as a negative correlation with the patient's age, dyslipidemia, and MPV value at the day when endovascular procedure was performed (Table 3). No MAE occurred in any of the patients.

\section{Discussion}

For this paper, we retrospectively analyzed the medical documentation of patients undergoing endovascular treatment due to lower extremity ischemia. The standard end-points were evaluated as the outcome measure using survival analysis. We found that within an approximately 1.5-year follow-up about $30 \%$ of the patients had required TLR or TER (about $70 \%$ primary stent patency). Such endovascular therapy outcomes corroborate the results of other authors. ${ }^{1,2}$

The loss of primary stent patency is an effect of in-stent restenosis, related mainly to neointima proliferation, and/ or atherosclerosis progression. In this study, TLR risk was significantly smaller in patients with dyslipidemia
(Table 1, Table 2, Fig. 1), greater BMI (borderline), and MPV (borderline), as well as in those with shorter aPTT and INR on the day of the first endovascular procedure (Table 2). Meanwhile, TER occurrence was related to the patient's age, LDL cholesterol blood concentration, hematocrit, and MPV, aPTT and INR values (Table 3 ).

The results obtained (Table 1-3, Fig. 1) seem to be inconsistent with the state of art knowledge concerning pathophysiology and the management of cardiovascular disorders, due to at least 2 reasons. Firstly, we did not show a statistically significant effect of diabetes (Fig. 2), smoking, and hypertension on the risk of TLR (Table 1, 2), and secondly, we found that dyslipidemia decreased an established end-point risk. However, it is known that elevated LDL cholesterol is recognized as one of the main traditional risk factors for cardiovascular diseases, atherosclerosis development and progression, and LDL cholesterol concentration below $70 \mathrm{mg} / \mathrm{dL}$, below $100 \mathrm{mg} / \mathrm{dL}$, and below $115 \mathrm{mg} / \mathrm{dL}$ are proposed as its target levels during therapy depending on individual patient risk stratification. ${ }^{16,17}$ Although risk factors for TLR in patients with lower limbs ischemia may be different than for coronary artery atherosclerosis progression, there is also some evidence that patients with continuing dyslipidemia had 
Table 3. The Cox proportional-hazards regression model for target extremity revascularization (TER). $\chi^{2}=39.44 ; p=0.013$

\begin{tabular}{|c|c|c|c|c|c|}
\hline Parameter & $\beta$-coefficient & $\beta 95 \% \mathrm{Cl}$ & $p$-value & $\begin{array}{l}\text { Hazard } \\
\text { ratio }\end{array}$ & $\begin{array}{c}\text { Hazard ratio } \\
95 \% \mathrm{Cl}\end{array}$ \\
\hline Age (years) & -0.069 & $-0.13--0.01$ & 0.02 & 0.93 & $0.88-0.99$ \\
\hline Smoking & -0.40 & $-1.25-0.46$ & 0.36 & 0.67 & $0.29-1.58$ \\
\hline Hypertension & 0.10 & $-0.85-1.06$ & 0.83 & 1.11 & $0.43-2.89$ \\
\hline Dyslipidemia & -3.08 & $-4.69--1.48$ & 0.0001 & 0.046 & $0.01-0.23$ \\
\hline $\mathrm{BMI}$ & -0.09 & $-0.26-0.08$ & 0.31 & 0.92 & $0.77-1.09$ \\
\hline History of CAD & -0.08 & $-1.19-1.02$ & 0.88 & 0.92 & $0.30-2.78$ \\
\hline Past stroke & -0.91 & $-2.56-0.74$ & 0.28 & 0.40 & $0.08-2.09$ \\
\hline History of $\mathrm{CHF}$ & -0.32 & $-2.01-1.37$ & 0.71 & 0.72 & $0.13-3.92$ \\
\hline LDL cholesterol (mg/dL) & 0.019 & $0.002-0.035$ & 0.024 & 1.02 & $1.00-1.04$ \\
\hline Triglycerides (mg/dL) & -0.002 & $-0.014-0.01$ & 0.75 & 0.99 & $0.99-1.01$ \\
\hline Leukocytes count (G/L) & 0.001 & $-0.08-0.08$ & 0.97 & 1.00 & $0.92-1.09$ \\
\hline Hematocrit (\%) & 0.18 & $0.02-0.35$ & 0.03 & 1.20 & $1.02-1.42$ \\
\hline $\mathrm{MCV}(\mathrm{fL})$ & -0.06 & $-0.17-0.05$ & 0.27 & 0.94 & $0.84-1.05$ \\
\hline Platelets count (G/L) & -0.002 & $-0.01-0.002$ & 0.28 & 0.99 & $0.99-1.00$ \\
\hline MPV & -0.42 & $-0.82--0.01$ & 0.045 & 0.66 & $0.44-0.99$ \\
\hline Creatinine (mg/dL) & 1.85 & $-1.06-4.75$ & 0.21 & 6.35 & $0.35-116.1$ \\
\hline INR & 0.46 & $0.12-0.79$ & 0.01 & 1.58 & $1.13-2.21$ \\
\hline aPTT & 0.16 & $0.07-0.26$ & 0.001 & 1.18 & $1.07-1.30$ \\
\hline Fast glucose (mg/dL) & -0.001 & $-0.02-0.02$ & 0.95 & 0.99 & $0.98-1.02$ \\
\hline
\end{tabular}

CI - confidence interval; BMI - body mass index; CAD - coronary artery disease; CHF - congestive heart failure; LDL - low-density lipoprotein; MCV - mean corpuscular volume; MPV - mean platelet volume; INR - international normalized ratio; aPTT - activated partial thromboplastin time.

worse outcomes after B, C and D classes of femoropopliteal lesion subintimal angioplasty with stenting. ${ }^{18,19}$ Moreover, aggressive therapy with statins in patients with PAD significantly reduced all-cause mortality and the risk of stroke and showed a trend towards decreasing the risk of other cardiovascular end-points. ${ }^{20}$ Statins also improved survival for 1 year after vascular surgery and demonstrated decreased incidence of lower extremity embolic complications after endovascular aortic repair. ${ }^{21,22}$ In addition, several studies in recent years have shown that statins improved pain-free walking distance, ankle-brachial index and treadmill exercise time. ${ }^{23,24}$ However, they did not affect long-term stent or bypass patency and limb salvage. ${ }^{25}$

In our study, a greater MPV value significantly decreased the risk of TER by approximately $34 \%$ (Table 3 ). In many investigations, an increase in MPV, but without a clearly defined cut-off value, was recognized as a marker of platelet renewal due to the baccelerated consumption in the thrombotic processes. ${ }^{26,27}$ Larger platelets may potentially take part in acute cardiovascular events, including peripheral artery disease, unprovoked deep vein thrombosis, and pulmonary embolism, as well as in chronic ISR processes (growth factors accelerate neointima proliferation). ${ }^{26-29}$ This data makes it difficult to explain our obser- vations, which showed a favorable effect of greater MPV on TER risk with an average HR of 0.66 (Table 3).

Abnormalities in plasma coagulation factors are recognized mainly as a risk for venous thrombo-embolic disease. However, our investigation showed that longer blood coagulation times (greater aPTT, and INR) result in increased risk of TLR and TER (Table 2, 3). This suggests that decrease in blood coagulation had paradoxically unfavorable effect on treatment outcomes in patients with PAD. The effect of blood coagulation disorders on the course of PAD has been investigated in the past, but without finding a significant effect on intermittent claudication, and in the prevention of restenosis/reocclusion following peripheral endovascular treatment. ${ }^{30,31}$

Being aged over 45 in males and 55 in females is recognized as a risk factor for cardiovascular event and death. ${ }^{16,17}$ In our study, paradoxically, older patients had a 7\% decreased risk of TER (Table 3). However, in a recent study by Dippel et al., increased age was also found to decrease the occurrence of TLR (HR 0.77; 95\% CI 0.61-0.96) when they compared the use of excimer laser atherectomy (ELA) with adjunctive percutaneous transluminal angioplasty (PTA) with PTA alone in a Cox proportional-hazards model. ${ }^{3}$ It is worth underlining that in 
this study, similar to our observations, diabetes mellitus was not an independent variable determining risk of TLR (Fig. 2). The negative correlation between age and TLR risk may be explained by greater atherosclerotic plaque stabilization, better collateral circulation development, and, possibly, a less invasive therapeutic attitude to older individuals.

As with most researchers authors, we could not avoid some methodological shortcomings that could have influenced the strength of the deductions based on our results. The main limitations of the paper are the retrospective type of analysis, the small sample size, the lack of inclusion of all peripheral artery stenting performed at our center, and the lack of a random selection of patients included in the analysis (potential selection bias). However, completion of these aforementioned requirements exceeded our abilities due to the great number of procedures introduced into our database (more than one thousand), and the lack of a complete follow-up for the treated patients. Moreover, the small number in the sample made it impossible to divide the patients according to the level of stenting performed, aortoiliac and femoropopliteal, which would certainly affect the risk of TLR and TER. ${ }^{2}$ On the other hand, the limitations mentioned did not interfere with our ability to achieve statistical significance in the analyses undertaken, although our results, especially concerning their lipidal aspects, should be interpreted carefully. On the other hand, any doubts that have arisen show the need to perform more studies on hypolipidemic medication regarding standard end-points in patients with PAD after stenting.

\section{Conclusions}

Endovascular treatment with stenting in patients with atherosclerotic peripheral arterial disease is effective (70\% of primary stent patency), especially as these procedures may be repeated and may postpone the need for a leg amputation.

Risk of TLR and TER was decreased to the greatest extent by the presence of dyslipidemia in spite of statins therapy.

The effect of dyslipidemia, hypolipidemic therapy, MPV, and main plasma coagulation tests (aPTT and INR) on the risk of TLR and TER need to be evaluated.

\section{References}

1. Laird JR, Yeo KK. The treatment of femoropopliteal in-stent restenosis: Back to the future. J Am Coll Cardiol. 2012;59:24-25.

2. Norgren L, Hiatt WR, Dormandy JA, et al. Inter-Society consensus for the management of peripheral arterial disease (TASC II). J Vasc Surg. 2007;45(Suppl S):S5-S67.

3. Dippel EJ, Makam P, Kovach R, et al. Randomized controlled study of excimer laser atherectomy for treatment of femoropopliteal instent restenosis: Initial results from the EXCITE ISR (EXCImer Laser Randomized Controlled Study for Treatment of FemoropopliTEal In-Stent Restenosis) Trial. JACC Cardiovasc Interv. 2015;8:92-101.
4. Bosiers $M$, Scheinert D, Peeters $P$, et al. Randomized comparison of everolimus-eluting versus bare-metal stents in patients with critical limb ischemia and infrapopliteal arterial occlusive disease. J Vasc Surg. 2012;55:390-398.

5. Singh GD, Armstrong EJ, Laird JR. Femoropopliteal in-stent restenosis: Current treatment strategies. J Cardiovasc Surg (Torino). 2014;55:325-33.

6. Speck U, Scheller B, Hamm B. Drug-coated balloons for restenosis prophylaxis. Rofo. 2014;186:348-358.

7. Schmidt $A$, Zeller $T$, Sievert $H$, et al. Photoablation using the turbo-booster and excimer laser for in-stent restenosis treatment: Twelve-month results from the PATENT study. J Endovasc Ther. 2014;21:52-60.

8. Liistro F, Grotti S, Porto I, et al. Drug-eluting balloon in peripheral intervention for the superficial femoral artery: The DEBATESFA randomized trial (drug eluting balloon in peripheral intervention for the superficial femoral artery). JACC Cardiovasc Interv. 2013; 6:1295-1302.

9. Tsetis D, Belli AM, Morgan R, et al. Preliminary experience with cutting balloon angioplasty for iliac artery in-stent restenosis. J Endovasc Ther. 2008;15:193-202.

10. Karthik S, Tuite DJ, Nicholson AA, et al. Cryoplasty for arterial restenosis. Eur J Vasc Endovasc Surg. 2007;33:40-43.

11. Tosaka A, Soga Y, lida $O$, et al. Classification and clinical impact of restenosis after femoropopliteal stenting. J Am Coll Cardiol. 2012;59: 16-23.

12. Swanson N, Hogrefe K, Javed Q, Malik N, Gershlick AH. Vascular endothelial growth factor (VEGF)-eluting stents: In vivo effects on thrombosis, endothelialization and intimal hyperplasia. $J$ Invasive Cardiol. 2003;15:688-692.

13. Grzelązka P, Koza K, Trofimiuk A, et al. The association between periprocedural factors and the late outcome of percutaneous stenting of lower extremity arteries. A retrospective cohort study. Postep Kardiol Inter. 2015;11(2);1-6.

14. Kavaliauskienè Z, Benetis R, Inčiūra D, Aleksynas N, Kaupas RS, Antuševas A. Factors affecting primary patency of stenting for TransAtlantic Inter-Society (TASC II) type B, C, and D iliac occlusive disease. Medicina (Kaunas). 2014;50:287-294.

15. Diehm N, Baumgartner I, Jaff M, et al. A call for uniform reporting standards in studies assessing endovascular treatment for chronic ischaemia of lower limb arteries. Eur Heart J. 2007;28:798-805.

16. Perk J, De Backer G, Gohlke H, et al.; European Association for Cardiovascular Prevention \& Rehabilitation (EACPR); ESC Committee for Practice Guidelines (CPG). European Guidelines on cardiovascular disease prevention in clinical practice (version 2012). The Fifth Joint Task Force of the European Society of Cardiology and Other Societies on Cardiovascular Disease Prevention in Clinical Practice (constituted by representatives of nine societies and by invited experts). Eur Heart J. 2012;33:1635-1701.

17. Montalescot G, Sechtem U, Achenbach S, et al. ESC guidelines on the management of stable coronary artery disease: The Task Force on the management of stable coronary artery disease of the European Society of Cardiology. Eur Heart J. 2013;34:2949-3003.

18. Gabrielli R, Rosati MS, Vitale S, et al. Randomized controlled trial of remote endarterectomy versus endovascular intervention for TransAtlantic Inter-Society Consensus II D femoropopliteal lesions. J Vasc Surg. 2012;56:1598-1605.

19. Baril DT, Marone LK, Kim J, Go MR, Chaer RA, Rhee RY. Outcomes of endovascular interventions for TASC II B and C femoropopliteal lesions. J Vasc Surg. 2008;48:627-633.

20. Antoniou GA, Fisher RK, Georgiadis GS, Antoniou SA, Torella F. Statin therapy in lower limb peripheral arterial disease: Systematic review and meta-analysis. Vascul Pharmacol. 2014;63:79-87.

21. Galiñanes EL, Reynolds S, Dombrovskiy VY, Vogel TR. The impact of preoperative statin therapy on open and endovascular abdominal aortic aneurysm repair outcomes. Vascular. 2014, pii: 1708538114552837.

22. Suckow BD, Kraiss LW, Schanzer A, et al. Statin therapy after infrainguinal bypass surgery for critical limb ischemia is associated with improved 5-year survival. J Vasc Surg. 2015;61:126-133.e1.

23. Markel A. Statins and peripheral arterial disease. Int Angiol. 2015;34: 416-427.

24. Poredoš $\mathrm{P}$, Jezovnik MK, Kalodiki Eet al. Medical management of patients with peripheral arterial disease. Int Angiol. 2015;34:75-93. 
25. Dosluoglu HH, Davari-Farid S, Pourafkari L, Harris LM, Nader ND. Statin use is associated with improved overall survival without affecting patency and limb salvage rates following open or endovascular revascularization. Vasc Med. 2014;19:86-93.

26. Leader A, Pereg D, Lishner M. Are platelet volume indices of clinical use? A multidisciplinary review. Ann Med. 2012;44:805-816.

27. Pal R, Bagarhatta R, Gulati S, Rathore M, Sharma N. Mean platelet volume in patients with acute coronary syndromes: A supportive diagnostic predictor. J Clin Diagn Res. 2014;8:MC01-4.

28. Taglieri N, Saia F, Rapezzi C, et al. Prognostic significance of mean platelet volume on admission in an unselected cohort of patients with non ST-segment elevation acute coronary syndrome. Thromb Haemost. 2011;106:132-140.
29. Seyyed-Mohammadzad MH, Eskandari R, Rezaei Y, et al. Prognostic value of mean platelet volume in patients undergoing elective percutaneous coronary intervention. Anadolu Kardiyol Derg. 2015;15:25-30.

30. Cosmi B, Conti E, Coccheri S. Anticoagulants (heparin, low molecular weight heparin and oral anticoagulants) for intermittent claudication. Cochrane Database Syst Rev. 2014;5:CD001999.

31. Robertson L, Ghouri MA, Kovacs F. Antiplatelet and anticoagulant drugs for prevention of restenosis/reocclusion following peripheral endovascular treatment. Cochrane Database Syst Rev. 2012;8:CD002071. 